\title{
The dynamics of ac Josephson effect in nematic liquid crystals
}

\author{
E. I. Kats and G. E. Volovik \\ LD Landau Institute for Theoretical Physics, Vorobjevskoe shosse 2, GSP-1 117940 Moscow V-334, USSR
}

(Reçu le 15 décembre 1978, révisé le 17 janvier 1979, accepté le 23 janvier 1979)

\begin{abstract}
Résumé. - On considère le mécanisme de glissement de phase par boucles de disclinations dans les cristaux liquides nématiques. On calcule la densité de disclinations à l'équilibre en fonction du gradient de vitesse angulaire appliqué et on étudie la dynamique macroscopique des nématiques en présence de disclinations.
\end{abstract}

\begin{abstract}
The disclination loop mechanism of phase slippage in nematics is considered. The equilibrium density of disclinations in terms of an applied gradient of the angular velocity is found. The macroscopic dynamics of the nematics with disclinations is discussed.
\end{abstract}

The ac Josephson effect has been intensively investigated in different systems with broken symmetry, such as superconductors, superfluid He II and superfluid phases of ${ }^{3} \mathrm{He}$. The phase slippage may occur in different ways depending on the topology of the space order parameter of the system, on the geometry of the sample, and on the boundary conditions, etc. For example in ${ }^{3} \mathrm{He}-\mathrm{A}$ the phase slippage may occur due to singular vortices as in He II [1], due to the coreless vortices [2] and due to a system of the solitons [3]. It is difficult to find out theoretically and experimentally what type of phase slippage takes place in different cases and to investigate the dynamics of this effect.

The liquid crystal may be a good model for investigations of this problem. The possibility of the ac Josephson effect in nematics was first considered by de Gennes [4]. He pointed out that there may be situations where the director $\mathbf{n}$ rotates with different angular velocities at different points of the vessel. In this case the deformation $\nabla_{i} n$ increases linearly in time, so the phase slippage is necessary to stop it. The first experimental evidence of the ac Josephson effect in nematics was made in MBBA [5] and was interpreted in terms of phase slippage in [6]. In [5] the layer of nematics with homeotropic boundary conditions was influenced by elliptically polarized shear flow in an external electric field.

In this paper we qualitatively consider a more simple geometry for the experiment, presuming the creation of the disclination loops to be the basic mechanism of phase slippage. We found that in this case the situation is quite analogous to the ac Josephson effect in He II in a capillary. For example, the dependence of equilibrium density of the singular lines on the applied gradient of the angular velocity $\nabla \omega$ is the same as the dependence of density of the singular vortices in $\mathrm{He}$ II on the applied gradient of the chemical potential $\nabla \mu[7,8]$. Investigating experimentally this dependence and the kinetics of the disclinations in the process of the dynamics of the ac Josephson effect in nematics one may make conclusions on the processes in the system of vortices in this effect in He II.

Let us consider the nematic between two parallel planes with the planar orientation of the director $\mathbf{n}$. The upper plane rotates with angular velocity $\omega$ with respect to the lower plane. If the length of the boundary viscous layer $d \sim(v / \omega)^{1 / 2}(v$ is kinematic viscosity) is larger than the distance $d$ between the planes, the equilibrium velocity field has vorticity linearly dependent on $z$ (the axis $z$ is perpendicular to the plane)

$$
\mathbf{v}=\omega \frac{z}{d} \hat{z} \times \mathbf{r}
$$

The director $\mathbf{n}$ also rotates, its motion is defined by the following equation

$$
K_{22} \frac{\partial^{2} \psi}{\partial z^{2}}=\gamma_{1}\left(\frac{\partial \psi}{\partial t}-\omega \frac{z}{d}\right)
$$

or

$$
K_{22} \frac{\partial^{3} \psi}{\partial z^{3}}=\gamma_{1}\left(\frac{\partial^{2} \psi}{\partial t \partial z}-\frac{\omega}{d}\right)
$$

Here $K_{22}$ is the Frank modulus, $\gamma_{1}$-coefficient of the rotational viscosity, $\psi$ defines the orientation of vector $\mathbf{n}$ in $x, y$ plane

$$
\mathbf{n}=\hat{x} \cos \psi+\hat{y} \sin \psi .
$$


It is easily seen that this equation has no solution with finite deformation $\partial \psi / \partial z$ (the solution $\psi=\omega z t / d$ has deformation $\partial \psi / \partial z=\omega t / d$ which is linear in time). Therefore we need some process of phase slippage which allows for deformation to relax. We consider the case

$K_{33}>2 K_{22}$ when it is energetically unfavourable for $\mathbf{n}$ to escape from the planar orientations. Therefore the basic mechanism for phase slippage is the creation of disclination loops and their turbulent motion in such a way, that the current of disclination is perpendicular to $\nabla \omega=\frac{\omega}{d} \hat{z}$ and equal to $\nabla \omega$ by its magnitude (compare with ac Josephson effect in He II where the current of vortices is equal to $\nabla \mu$ by its magnitude).

The main features of this turbulent motion may be found from dimensional analysis by the following consideration. Given $z_{0}$ to be the average distance between the disclinations and $u_{0}$ their velocity with respect to the liquid, the density of disclinations, is proportional to the length of the singular lines per unit volume,

$$
n \sim \frac{1}{z_{0}^{2}} .
$$

The disclination current $n u_{0} \sim u_{0} / z_{0}^{2}$ must be equal to the gradient of the angular velocity. So we have the first relation

$$
\frac{u_{0}}{z_{0}^{2}} \sim|\nabla \omega|
$$

Considering the isolated line in its steady motion one may derive the Magnus force per unit length acting on the line from deformation field $\nabla \psi$

$$
F_{\text {magn }} \sim K_{22} \frac{\partial \psi}{\partial z} \sim K_{22} / z_{0}
$$

must be compensated by the friction force acting from the liquid $F_{\mathrm{fr}} \sim \gamma_{1} u_{0}$. So we have the second relation

$$
\gamma_{1} u_{0} \sim \frac{K_{22}}{z_{0}}
$$

From these two relations (4) and (5) one may find $z_{0}$

$$
z_{0} \sim\left(\frac{K_{22}}{\gamma_{1}|\nabla \omega|}\right)^{1 / 3}, n \sim\left(\frac{\gamma_{1}|\nabla \omega|}{K_{22}}\right)^{2 / 3} .
$$

We may see that this result corresponds to the fact that in the turbulent regime all the terms in eq. (2a) are equal to each other.

The turbulent motion is also characterized by the equilibrium deformation $\langle\nabla \psi\rangle$, which depends on $\nabla \omega$ in the following way

$$
\nabla \omega \sim \frac{K_{22}}{\gamma_{1}}\langle\nabla \psi\rangle .|\langle\nabla \psi\rangle|^{2} .
$$

The same law is valid for He II, where the equilibrium deformation is the superfluid velocity $\mathbf{v}_{\mathbf{s}}$. In this case eq. (7) has the form

$$
\nabla \mu \sim\left(v_{s}-v\right)\left(v_{s}-v\right)^{2}
$$

here $\mathbf{v}$ is the velocity of the normal component.

The equation (7) may be also interpreted in terms of the disclination current. The eq. (7) means that the dissipative current of disclinations is proportional to the cube of deformation.

We may estimate dissipation connected with motion of disclinations as follows :

$$
R \sim \nabla \omega . \mathcal{F}_{\text {magn }} \sim \frac{K^{2}}{\gamma_{1}}|\nabla \psi|^{4} .
$$

Compare with He II, where

$$
R \sim|\lambda|^{4}, \quad \lambda=\frac{\partial E}{\partial v_{\mathrm{s}}}=\rho_{\mathrm{s}}\left(\mathbf{v}_{\mathrm{s}}-\mathbf{v}\right) .
$$

The relations (6), (7) and (9) may be experimentally verified in nematics. For example the density of the singular lines and their velocities of motion may be investigated visually.

We thank I. E. Dzyaloshinskii and V. P. Mineyev for valuable discussions and L. M. Blinov who acquainted us with papers $[5,6]$.

\section{References}

[1] Anderson, P. W., Rev. Mod. Phys. 38 (1966) 298.

[2] Anderson, P. W., Toulouse, G., Phys. Rev. Lett. 36 (1976) 594.

[3] VoloviK, G. E., Zh. ETF pis'ma 27 (1978) 605 ; Hook, J. R., Hall, H. E., Submitted to J. Phys. C.

[4] De Gennes, P. G., The Physics of Liquid Crystals (Clarendon Press, Oxford) 1974.

[5] Pieranski, P., Guyon, E., Phys. Rev. Lett. 39 (1977) 1280.
[6] Dreyfus, J. M., Pieranski, P., Guyon, E., Phase slippage and hydrodynamic instabilities obtained in a nematic layer submitted to an elliptically polarized flow, Preprint.

[7] Vinen, W. F., Proc. R. Soc. A 240 (1957) 114, 128 ; 242 (1957) $493 ; 243$ (1958) 400.

[8] Schwarz, K. N., Phys. Rev. B 18 (1978) 245. 